\title{
Sistemas de Polinúcleos Sostenibles (SPS). Estrategias de Redensificación de Desarrollos Urbanos Lineales: La Manga del Mar Menor.
}

\author{
Enrique Mínguez Martínez ${ }^{12}$, \\ David Maestre Celdrán ${ }^{3}$, Ana Isabel Doménech García ${ }^{4}$ \\ ${ }^{1}$ Departamento de Edificación y Urbanismo, Universidad de Alicante. Alicante, España, \\ ${ }^{12}$ Departamento de Ciencias Politécnicas. Universidad Católica San Antonio. Murcia, España, \\ ${ }^{3}$ Enrique Mínguez Arquitectos \\ E-mail: 'enrique.minguez@ua.es, ${ }^{2}$ enrique.minguez@ua.es, \\ 33maestre@ucam.edu, ${ }^{3}$ eminguez@eminguez.com
}

\begin{abstract}
Resumen. El futuro de nuestras ciudades se determinará por los procesos de regeneración y mejora del tejido urbano, proponiendo nuevos patrones que favorezcan una transformación hacia nuevos modelos urbanos equilibrados. "La ciudad compacta, multicéntrica, de usos mixtos, que favorece el andar, ir en bicicleta y el transporte público, es la forma urbana más sostenible. Una ciudad compacta, conectada y bien diseñada es una estructura flexible que relaciona las partes con el todo» (Rogers, 1999). Para conseguir estos objetivos, es necesario desarrollar nuevos modelos de planificación que permitan coordinar y analizar los diferentes factores de influencia urbana. El Sistema de Polinúcleos Sostenibles consigue, apoyándose en distintas estrategias urbanas pasivas, conformar una herramienta que permite un análisis comparativo de las distintas opciones de mejora para un entorno, contrastando su posible repercusión en la regeneración urbana, facilitando el camino hacia una nueva ciudad compacta. «La tarea es promover la vida urbana de los ciudadanos, alojados - esperémoslo - en concentraciones lo bastante densas y diversas como para ofrecerles una sólida oportunidad de desarrollar la vida urbana. » (Jacobs, 2011). La Manga del Mar Menor, entorno con un incuestionable atractivo paisajístico y climático, presenta un desarrollo urbanistico desordenado basado en una explotación económica inmediata. La estrategia de redensificación polinuclear aplicada a la Manga del Mar Menor, supondría la oportunidad de una recuperación urbana, corrigiendo los defectos del modelo de crecimiento actúal, así como crear las condiciones propicias para un tipo de desarrollo más sostenible e integrador tanto a nivel físico como social, garantizando la supervivencia del ecosistema.
\end{abstract}

Palabras clave: Sistema de Polinúcleos Sostenibles (SPS), la Manga del Mar Menor, ciudad compacta, redensificación, ciudad difusa.

\begin{abstract}
Sistema de Polinúcleos Sostenibles (SPS).
Los crecimientos de ciudades densas y compactas pueden operar a favor de la sostenibilidad entre otras razones, por el menor consumo de suelo, la reducción de los costes de las infraestructuras, la posible reducción de
\end{abstract}

emisiones y el ahorro energético, siempre que la planificación responda a los requerimientos de sus ciudadanos e incluya de un modo integrado estos parámetros en el diseño urbano. Más allá de una densidad meramente constructiva, es necesario garantizar la masa crítica imprescindible para dotar de vida a las 
ciudades «la ciudad es un receptáculo para la vida» (Alexander, 1968).

El Sistema de Polinúcleos Sostenibles (SPS) persigue este objetivo, posibilitando articular territorios de cualquier escala, al organizar la ciudad en núcleos conectados entre sí mediante redes de transporte público eficaces que constituyen una entidad urbana de mayor tamaño. La ciudad proyectada, compacta y diversa, conforma una red de barrios con sus propios parques y espacios públicos integrando toda una variedad de actividades públicas y privadas, donde las personas la habiten en la máxima extensión de la palabra. «La gente es el principal recurso de un territorio inteligente» (Vegara, de Las Rivas, 2004).

Mientras que la zonificación por actividades conduce a una mayor dependencia del coche privado, los nodos compactos del SPS reducen los desplazamientos en automóvil aumentando el uso de la bicicleta y la circulación peatonal. «Los nodos compactos de uso mixto disminuyen las necesidades de desplazamiento y generan unos bulliciosos barrios sostenibles» (Rogers, Gumuchdjian, 2000).

\section{La Manga de Bonet Castellana.}

En 1960 Antonio Bonet Castellana junto a Josep Puig Torné reciben el encargo de Tomás Maestre, dueño de la mayor parte de los terrenos de la Manga, de acometer el Plan de Ordenación y Urbanización de la Manga del Mar Menor (Fig. 1).

Antes de acometer la Ordenación de la Manga del Mar Menor, Bonet había adquirido gran experiencia en la elaboración de planes urbanísticos. En su etapa de estudiante asiste al desarrollo del Plan de Buenos Aires (1938) realizado por Le Corbusier en colaboración con Ferrari Hardoy y Juan Kurchan, con los que Bonet formaría el Grupo Austral en Buenos Aires cuando se exilia a Sudamérica.

En el Conjunto Urbanístico Casa Amarilla proyectado en Buenos Aires en 1943, Bonet plantea una reinterpretación del Plan de Buenos Aires con la inserción de un trozo de ville radieuse. En la propuesta, ya aparece uno de los principios directores que le seguirán en proyectos posteriores como la densificación del centro "(...) Llegaremos a reducir las ciudades, al conseguir densidades muy superiores a

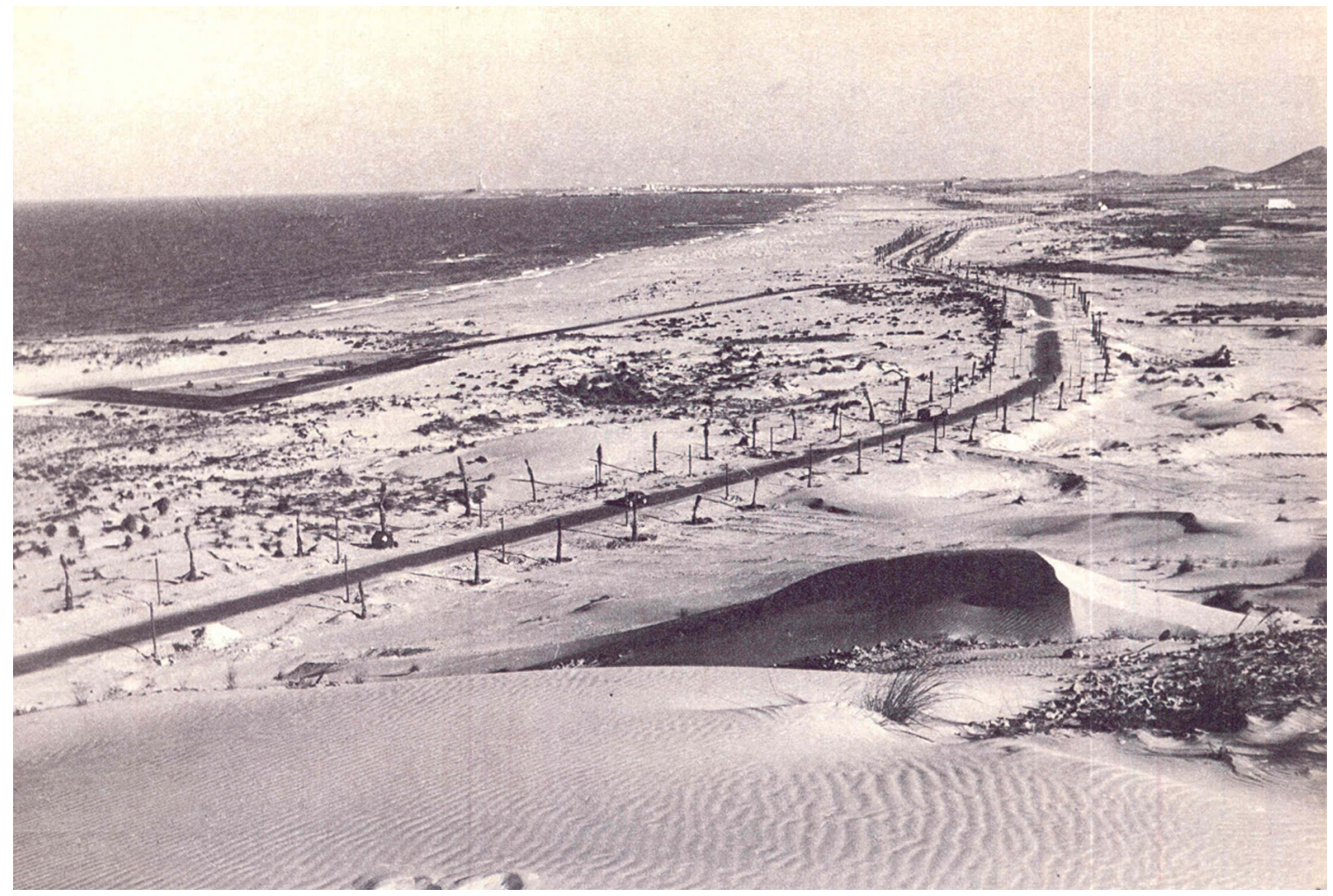

Fig. 1. Duna infinita entre dos mares. La Manga del Mar Menor.

(Fuente: Archivo histórico del Colegio de Arquitectos de Cataluña) 


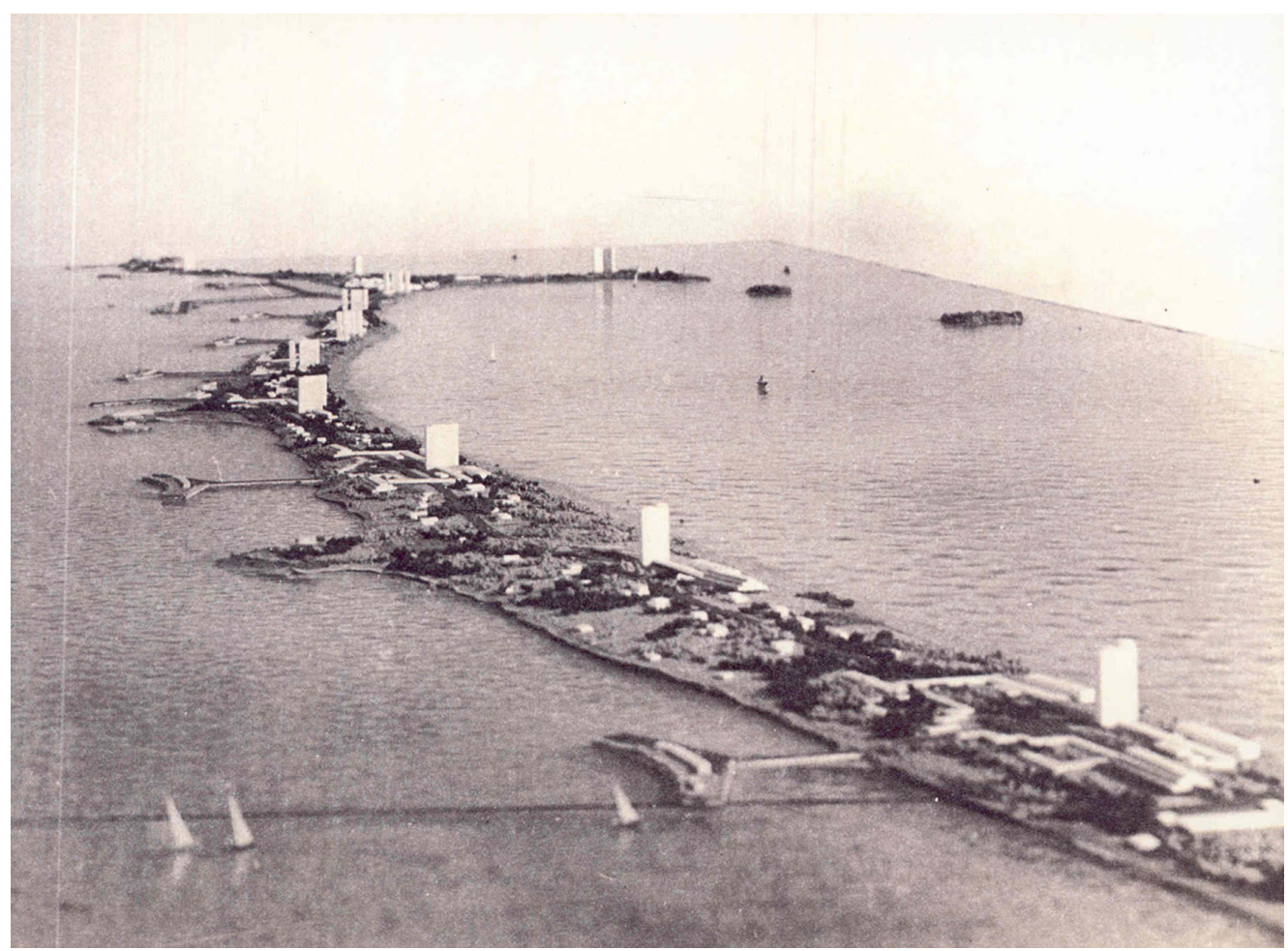

Fig. 2. Fotografía de la maqueta de la Ordenación de La Manga del Mar Menor. 1962. (Fuente: Archivo histórico del Colegio de Arquitectos de Cataluña)

las actuales, ocupando la mínima parte de la superficie de la ciudad (...)" Bonet, 1943 (citado en Álvarez, Roig, 1996). Principio que planteará en el proyecto de Ordenación de la Manga del Mar Menor (1960) y en sus dos propuestas urbanísticas más importantes en Barcelona, el Plan de Montjuic (1964) con Bohigas y Martorell y el Plan de la Ribera (1964), donde la densificación adquiere un papel preponderante frente a la dispersión.

La propuesta inicial para la Manga era un Plan equilibrado que nace de la lectura del lugar. La idea básica consiste en la creación de una serie de agrupaciones urbanas autónomas definidas por un cuerpo alto y edificaciones más bajas, creando un contrapunto con la estrecha franja de tierra de más de $22 \mathrm{Km}$ de largo sobre el Mar Menor y el Mediterráneo.

El proyecto inicial de Bonet y Puig muestra una intensa preocupación por el lugar y el territorio. Se observa como a lo largo de la Manga ponen en valor como aspecto estructurante del proyecto:

- Contrarrestar la horizontalidad del paisaje con la presencia de edificaciones en altura (grupo de torres) de manera espaciada.
La ordenación inicial se basa en la repetición de núcleos compactos prácticamente idénticos de densidades similares (12 núcleos para 2500 personas, denominados poblados turísticos) y separados $1 \mathrm{Km}$ uno de otro. En cada núcleo se proyecta una isla artificial y un grupo de torres de veinte plantas para hoteles, viviendas o apartamentos con piscina, lavandería, jardines, sumando un total de 48 torres (Fig. 2). "El terreno actual solo tiene una dimensión: al largo. Con las torres sistemáticamente dispuestas y las islas artificiales le añadimos dos nuevas dimensiones: alto y ancho" (Bonet, 1962).

Las torres, tal y como expresa Llobet i Ribero en "Hilberseimer y Mies. La metrópoli como ciudad jardín", son acentos verticales que se elevan hacia el cielo. Son el testimonio de la concentración y garantizan las ventajas de la vida urbana definiendo el espacio sin encerrarlo. La verticalidad de las torres como hitos paisajísticos, apoyados en una plataforma sobre la que se recorta el paisaje natural, es una estrategia similar a la ensayada por Adalberto Libera en su propuesta del Concurso de Ordenación para el Litoral de Castelfusano 
(Roma) entre los años 1933-34.

"El futuro será del planeamiento de tres dimensiones, en el que los edificios no lo serán en el sentido tradicional de la palabra, sino fragmentos de ciudad" Bonet, 1949 (citado en Álvarez, Roig, 1996).

El Plan de Ordenación de la Manga del Mar Menor de Bonet preservaba intacto gran parte del territorio, concentrando la edificación en núcleos complejos que se asemejan al modelo de ciudad compacta. "Las ciudades densas pueden diseñarse mediante una planificación integradora con el fin de aumentar el rendimiento energético, consumir menos recursos, producir menos polución y evitar expandirse sobre el paisaje rural. Por esas razones, creo que deberíamos profundizar en la idea de "ciudad compacta" - una ciudad densa y socialmente diversa - donde las actividades sociales y económicas se solapen y donde las comunidades puedan integrarse en su vecindario" (Rogers, Gumuchdjian, 2000).

Cada unidad vecinal era independiente, consiguiendo un entorno urbano complejo, con la suficiente masa crítica para lograr espacios confortables y una densidad equilibrada. "Las construcciones altas, situadas a gran distancia unas de otras, deben liberar el suelo en favor de grandes superficies verdes. (...). La densidad de su población debe ser lo suficientemente alta para dar validez a la disposición de instalaciones colectivas que sean una prolongación de las viviendas." (Carta de Atenas, 1931).

La propuesta inicial para la Manga era una planificación del asentamiento del hombre en la naturaleza, transformándola sin que ésta pierda su propia esencia. Un desarrollo turístico diseñado para crecer y desarrollarse a lo largo del tiempo, fomentando la sostenibilidad del turismo y la conservación del paisaje.

Los núcleos compactos y complejos proyectados, aunque se desarrollan de manera autónoma, están estrechamente conectados a través de la red viaria y las unidades de paisaje, caracterizados por conjuntos de viviendas unifamiliares con parcelas cada una de ellas de $1 / 2$ ha.

Sin embargo, conforme avanza la urbanización de La Manga, la parcelación inicial de viviendas unifamiliares en parcelas de $5.000 \mathrm{~m} 2$ se reduce considerablemente a parcelas de $1.000 \mathrm{~m} 2$ y $2.000 \mathrm{~m} 2$ (hasta cinco veces más pequeñas).

Esta fragmentación generó mayor superficie de viario secundario y la transformación de las unidades del paisaje entre los núcleos urbanos proyectados.

\section{Aplicación del Sistema de Polinúcleos Sostenibles (SPS).}

Según datos de la Agencia de Ecología Urbana de Barcelona (AEUB) reflejados en la Guía Metodológica (Rueda, 2012), para un ámbito del tamaño de un núcleo (entre Unidad Urbana y Barrio), en un tejido existente, la densidad neta recomendada oscila entre 100 y 150 viviendas por hectárea para una altura media de planta baja y cuatro alturas. Las densidades medias y medio-altas acortan las distancias, permiten trayectos peatonales, favorecen la implantación del transporte público y reducen el uso del vehículo privado. En el libro Ciudades para la gente (Gehl, 2014), se indica que, para nuevas áreas residenciales de alta densidad, 155 viviendas por hectárea es la relación adecuada.

Jane Jacobs sugería que 250 viviendas por hectárea era una condición necesaria para una ciudad viva y participativa. Autores como Hall (1999), Florida (2002), Jenks (1996), Lozano (1990), Newman \& Kenworth (1999), Urhahn (1996), van Kann \& Leduc (2008) apuestan por las altas densidades y la ciudad compacta como requisitos para conseguir entornos sostenibles.

"Crecemos y crecemos con parámetros de baja densidad, con esos monotemáticos chalets endosados o emparedados que nos esclavizan de por vida al vehículo privado"(Alonso, 2010). Además, el modelo disperso que se genera es de poco interés paisajístico, arquitectónicamente monótono y energéticamente insostenible.

"No se puede construir ciudad con porcentajes de suelo destinado a los usos básicos (vivienda) que, con frecuencia, apenas alcanzan el $30-35 \%$ de sus respectivos sectores, siendo el resto espacios destinados a viario, zonas verdes y libres públicas o equipamientos extensivos" (López de Lucio, 2007).

El Modelo denominado Polinúcleo Urbano Sostenible está caracterizado porque la suma de los suelos destinados a residencial, 


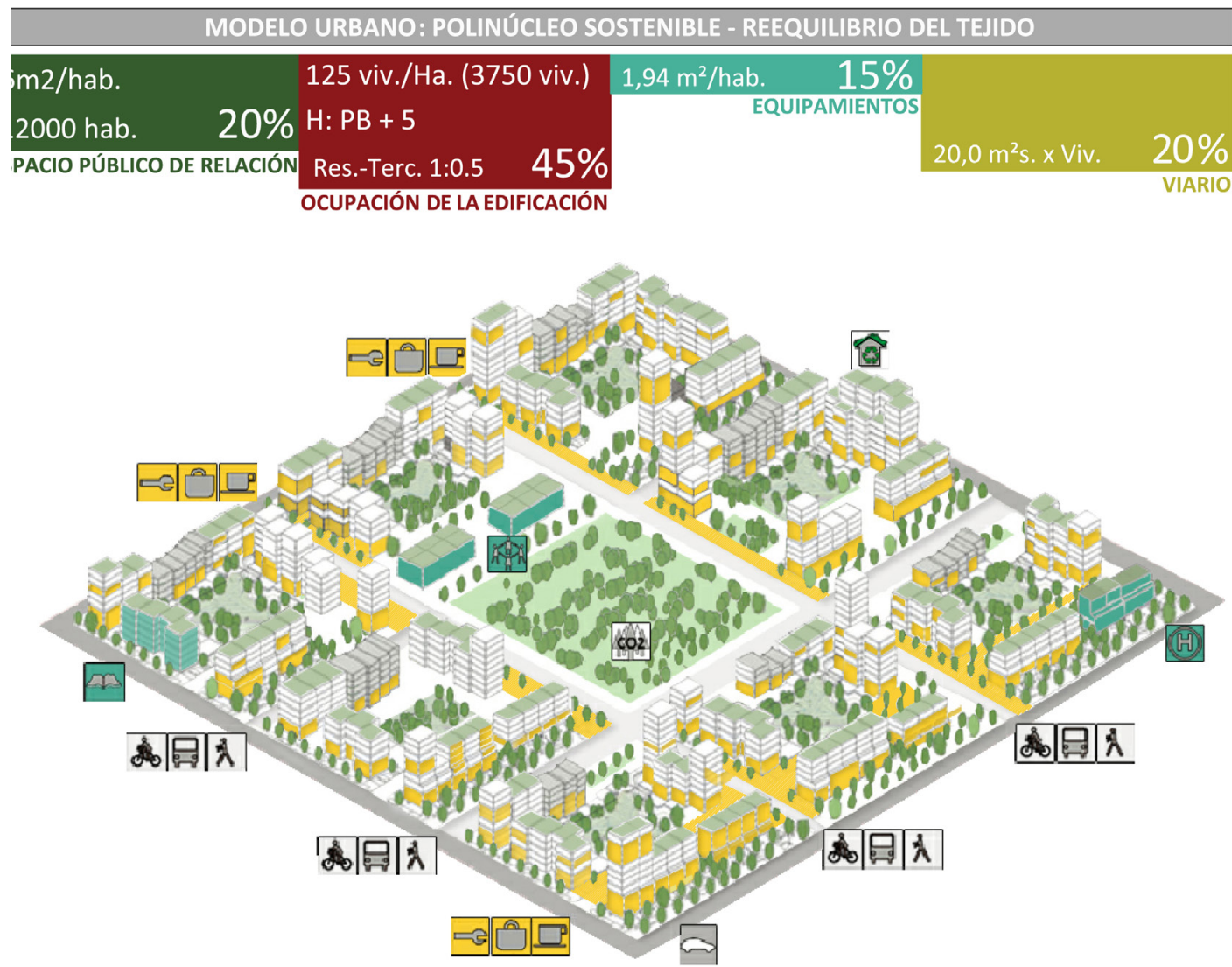

Fig. 3. Reparto del suelo de un Polinúcleo Sostenible.

Supermanzana eficiente. (Fuente: Elaboración propia, 2015)

equipamientos y uso terciario supera la mitad de la superficie total del sector, lo que nos permite crear una estructura urbana razonablemente densa, compacta y continua, materializada mediante manzanas híbridas (Fig. 3).

Siguiendo la metodología del SPS, y, dadas las singularidades geográficas de la Manga del Mar Menor, podemos establecer una serie de ámbitos con unas características similares, donde es viable la implantación de diferentes núcleos denominados Áreas de Identidad Homogénea (AIH). Estas áreas se han ubicado en las zonas de mayor intensidad (núcleos de mayor densidad y compacidad) para posibilitar la concentración de las actividades, acortando las distancias para facilitar los desplazamientos a pie y mejorando la movilidad. De esta manera, creamos una unidad vecinal autosuficiente en cada Área de Identidad Homogénea (Fig. 4).

Estas Áreas de Identidad Homogénea vienen definidas fundamentalmente por el equilibrio entre dos parámetros, la densidad y su tamaño.
"Para generar una diversidad exuberante en las calles y distritos de una ciudad (...) ha de haber también una concentración humana suficientemente densa, sean cuales fueren los motivos que los lleve allí." (Jacobs, 2011).

En el caso de la Manga del Mar Menor, podemos reactivar este espacio reequilibrando la estructura urbana existente y adaptándola a un Sistema de Polinúcleos Sostenibles (SPS) con un diámetro de 850 metros centrados en las zonas de mayor concentración urbana y una separación entre éstos que oscila entre los 200 y los 350 metros.

Se desarrollarán los Polinucleos Sostenibles en las áreas de mayor concentración edificatoria, potenciando medidas que permitan una futura liberalización del territorio. Este territorio es absolutamente lineal por lo que, aunque el diámetro máximo del Polinúcleo Sostenible sea de $850 \mathrm{~m}$, el ancho medio aproximado es de unos $400 \mathrm{~m}$ con lo que su superficie media alcanza las 30 has. Con estas dimensiones (850 

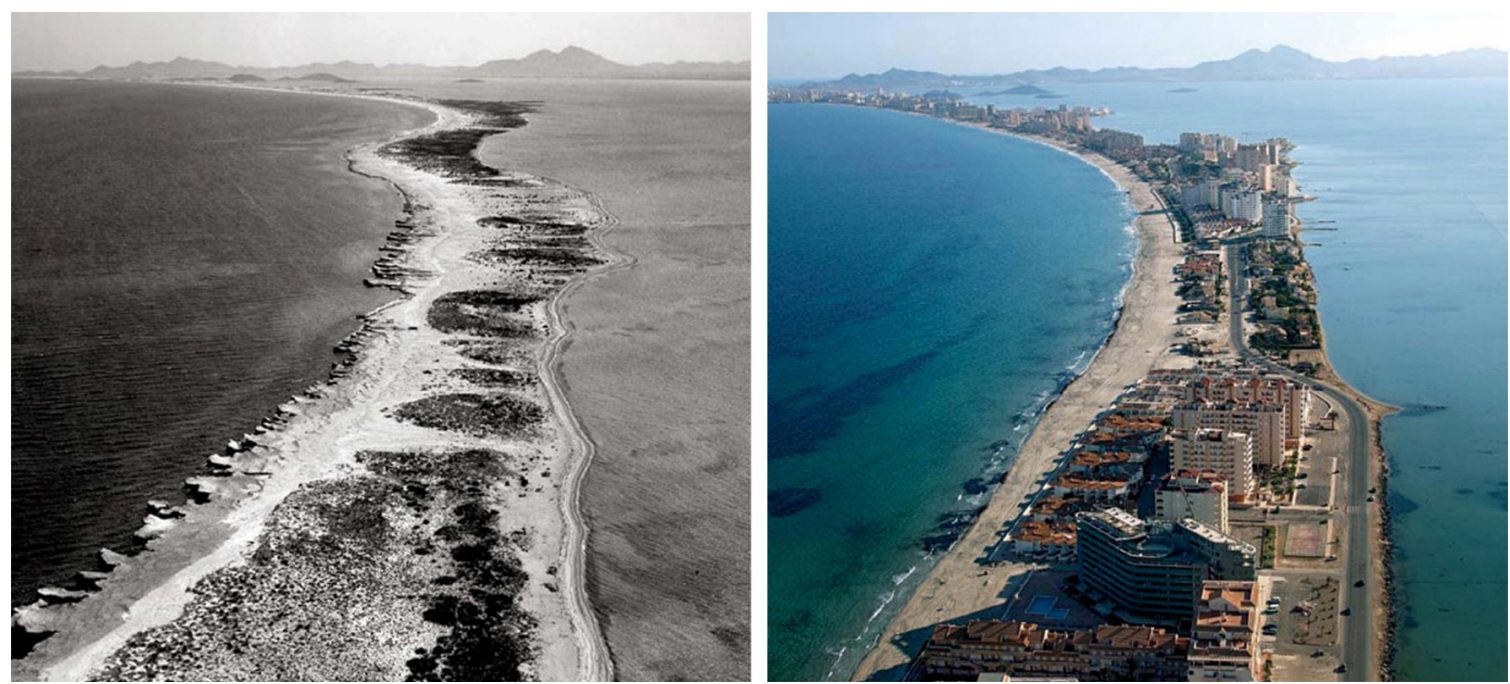

Fig. 4. Evolución de La Manga del Mar Menor (1930-2018). (Fuente: Región de Murcia Digital)

m de longitud máxima y 300 m de separación entre "barrios"), conseguimos reorganizar la movilidad, fortalecer el papel del peatón, crear las conexiones necesarias para desarrollar la vida sin necesidad del automóvil y contar con un transporte público eficiente.

\section{Medidas de Reequilibrio del Tejido. Liberalización del Espacio Natural.}

En la actualidad, el tejido de la Manga cuenta con una estructura desequilibrada y desorganizada. Ámbitos donde únicamente existen viviendas unifamiliares con una densidad media de 10 viv/ha, y zonas de grandes núcleos urbanos, quizá herencia del Plan Bonet, con densidades medias altas.

Se pueden reconocer ritmos edificatorios conformados en una serie de intervalos en el territorio que, en una secuencia continua, intercalan "núcleos" de una densidad superior a $70 \mathrm{viv} / \mathrm{ha}$ con otros de densidades inferiores a $10 \mathrm{viv} / \mathrm{ha}$ de una manera intermitente. Esto posibilita, a priori, establecer una estrategia clara de análisis al contar con dos tipologías características que se repiten: viviendas unifamiliares aisladas y edificación en bloque.

Dado que la estructura alta densidad - baja densidad, se repite a lo largo del territorio, es viable desarrollar un procedimiento tipo que posibilite establecer conclusiones contrastadas. Partiendo del objetivo de plantear pasillos visuales en las áreas apenas construidas, la estrategia consiste en reequilibrar y compactar (redensificar) las áreas de mayor densidad trasladando la edificabilidad de las viviendas unifamiliares a los grandes núcleos urbanos y de esta manera potenciar la liberalización del espacio natural. Recuperar la idea de vertical sobre horizontal y, transversal sobre longitudinal, clarificando y equilibrando la lectura del lugar tal y como reflejaban en sus primeras ideas básicas Bonet y Puig Torné "Plan de torres que ligan el conjunto y le dan verticalidad al paisaje".

En el caso de la Manga se han seleccionado indicadores que nos permiten testar los índices de sostenibilidad de los polinúcleos marcados en la figura 5, y por lo tanto precisar las estrategias a adoptar para cada problemática.

\section{Indicador: Densidad.}

Con el aumento de la densidad edificatoria (ciudad densa) se consigue liberalizar el territorio y concentrar la población suficiente para reactivar el tejido urbano.

Resulta imprescindible completar el concepto de densidad con otros como la ocupación o las alturas medias para obtener datos que reflejen las características del tejido analizado.

La lectura que nos aporta la densidad sobre el tipo de tejido urbano existente es parcial pero muy valiosa. Nos proporciona datos inmediatos para evaluar el grado de compactación de la 


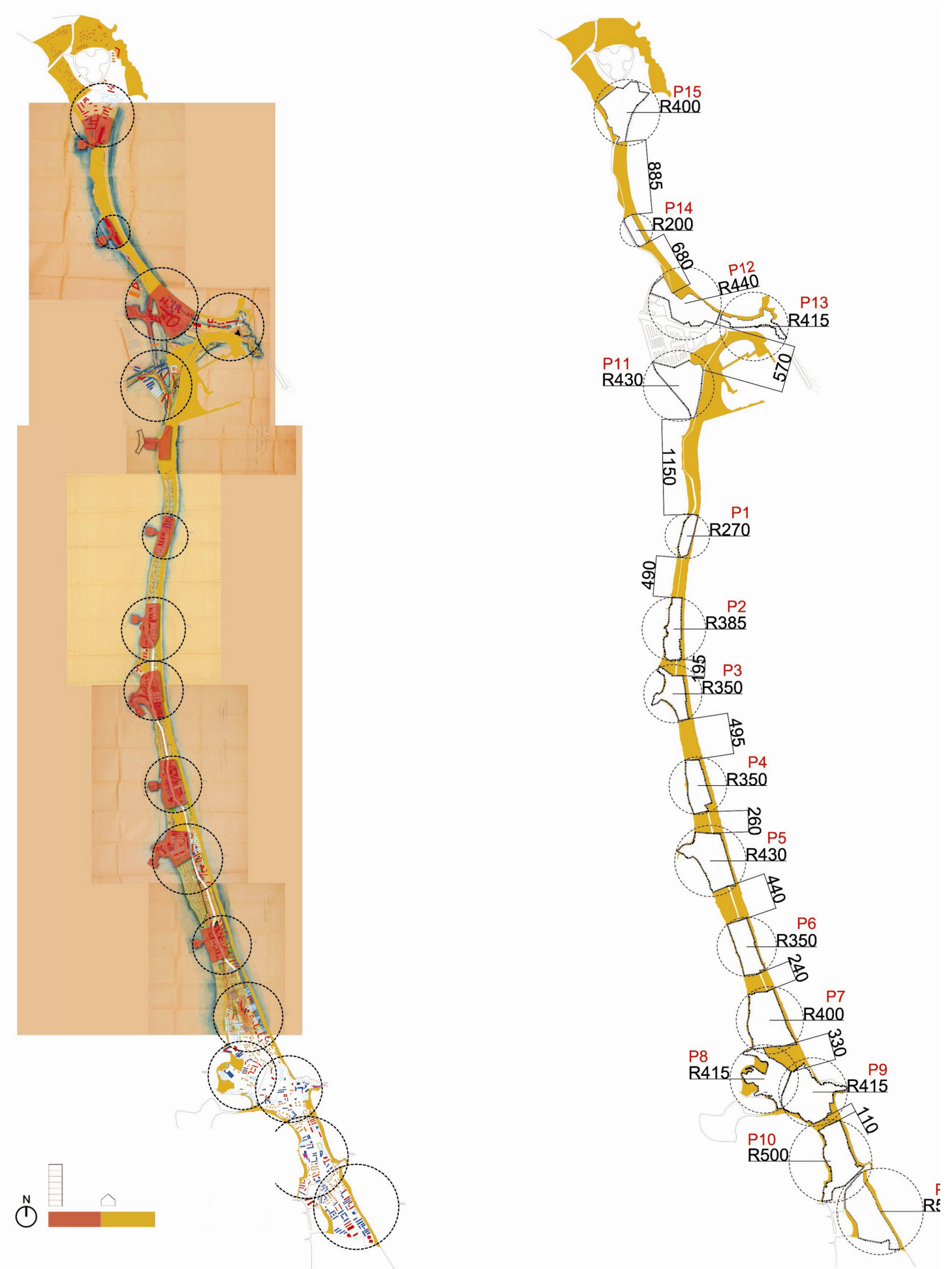

Fig. 5. Superposición de la ordenación proyectada por Bonet sobre la estructura urbana actual (izda.). Intensidad edificatoria y distancias entre los polinúcleos existentes en la Manga del Mar Menor (dcha.). (Fuente: Elaboración propia) 


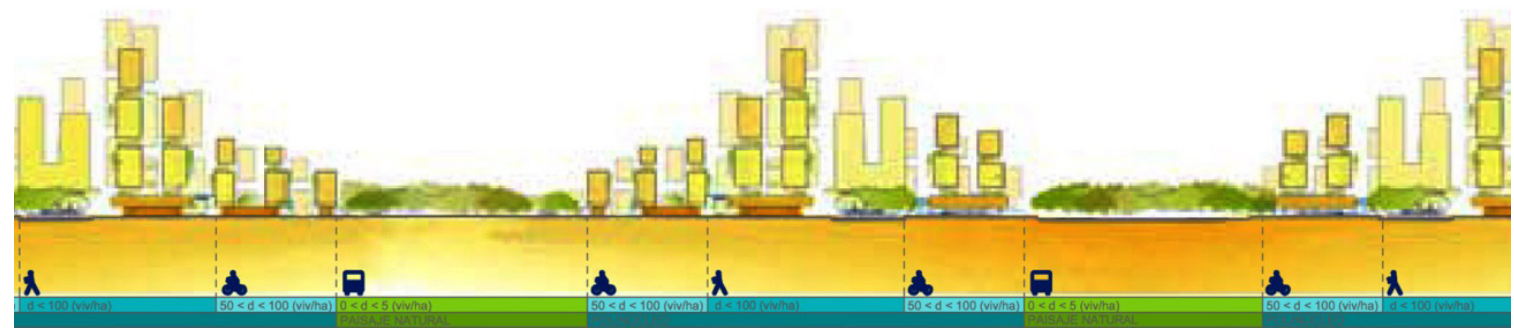

Fig. 6. Sección conceptual Sistema de Polinúcleos Sostenibles (SPS). (Fuente: Elaboración Propia)

ciudad y así dar el primer paso para lograr un entorno sostenible. "La densidad de viviendas es una variable directamente derivada del modelo de ocupación del territorio. La ciudad compacta debe ser la estrategia a seguir, conteniendo en la medida de lo posible el consumo de suelo. La densidad de viviendas es el primer condicionante que determina la proximidad de las personas a las funciones propias de la vida urbana: el contacto con otras personas, servicios, transporte, actividad económica, etc.” (Rueda, 2012).

\section{Indicador: Liberalización del espacio natural.}

Nos encontramos con un tejido muy heterogéneo, en realidad existen zonas puntuales muy compactas frente a otras muy dispersas.

El primer paso debería ser plantear una flexibilización de la normativa, permitiendo diferentes usos, más allá de los turísticos o residenciales.

Además de la flexibilización de los usos del suelo, se propone concentrar la edificabilidad para dejar sin construir parcelas con valores naturales o en primera línea de playa, tal y como se recoge en la Evaluación Ambiental Estratégica del Plan General Municipal de Ordenación Urbana (PGMO) de San Javier.

Estas estrategias, incrementarían el atractivo del paisaje, permitiendo una ordenación más flexible del territorio (Fig. 6).

Se propone en aquellas zonas donde la densidad es inferior a $10 \mathrm{viv} / \mathrm{ha}$, transferir dicha edificabilidad a los núcleos adyacentes.

Lo interesante del proceso de trasladar la edificabilidad, es el mecanismo que permite liberar un territorio con tanto potencial paisajístico

Para que este proceso se desarrolle, hay que modificar el planeamiento en la línea ya iniciada por el Ayuntamiento de San Javier, distribuyendo las edificabilidades.

\section{Conclusiones}

El Sistema de Polinúcleos Sostenibles (SPS) es una metodología que permite ordenar y clasificar los conceptos a aplicar sobre un entorno urbano para su revitalización, así como establecer unos valores patrón que faciliten una evolución hacia la ciudad compacta.

Partiendo de un análisis exhaustivo mediante Indicadores Individuales de Sostenibilidad Urbana (densidad edificatoria, compacidad absoluta, compacidad corregida, liberalización del paisaje, ...) se puede obtener una visión global de las fortalezas y debilidades del entorno de análisis, que facilita la propuesta, control y evaluación de las operaciones de regeneración.

Para conseguir que el reequilibrio del tejido se materialice es necesario distribuir las edificabilidades de modo que se concentren en las áreas de mayor densidad además de ofrecer estímulos económicos (o permutas) para que progresivamente las áreas de menor densidad vayan liberando el paisaje natural.

\section{Referencias}

Alexander, Christopher. 1968. "La Ciudad no es un árbol". En Cuadernos Suma-Nueva Visión 9, 20-30. Buenos Aires: Lala Méndez Mosquera, arq. y Jorge Grisetti.

Alonso, Luis. 2010. 40 ideas para 100 proyectos. Barcelona: Loft Publications.

Álvarez, Fernando., y Roig, Jordi. 1999. 
Bonet Castellana. Barcelona: Santa Cole y Escuela Técnica Superior de Arquitectura de Barcelona.

Gehl, Jan. 2014: Ciudades para la gente. Buenos Aires: Infinito

Jacobs, Jane. 2011. Muerte y vida de las grandes ciudades. Madrid: Capitán Swing Libros, S.L.

López de Lucio, R. 2007. Construir Ciudad en la Periferia. Criterios de Diseño para Áreas Residenciales Sostenibles. Madrid: Mairea Libros.

Llobet, Xavier. 2007. Hilberseimer y Mies. La metrópoli como ciudad jardín. Barcelona: Fundación Caja de Arquitectos.

Rogers, Richard (dir). 1999. Towards an Urban Renaissance. Londres: Urban Task Force.

Rogers, Richard, y Gumuchdjian, Philip. 2000. Ciudades para un pequeño planeta. Barcelona: Gustavo Gili.

Rueda, Salvador (dir). 2012. Guía Metodológica para los sistemas de auditoría, certificación o acreditación de la calidad y sostenibilidad en el medio urbano. Madrid: Centro de Publicaciones Secretaria General Técnica. Ministerio de Fomento.

Vegara, Agustín, y De Las Rivas, Juan Luis. 2004. Territorios Inteligentes. Madrid: Fundación Metrópoli. 